\title{
Radiology of COVID-19 pneumonia
}

\section{Qonita Said-Hartley and Sulaiman M oosa}

Division of Radiology, Department of Medicine, Faculty of Health Sciences, University of Cape Town and Groote Schuur Hospital, Observatory, Cape Town, South Africa

Address for correspondence:

Dr Q. Said-Hartley

Division of Radiology

Room 16, Cl6

Groote Schuur Hospital

Observatory

7925

South Africa

Email:

qonita.said-hartley@uct.ac.za

\section{BACKGROUND}

Coronavirus disease (COVID-19) was declared when a cluster of pneumonia cases was reported in Wuhan, China, on 31 December 2019. The causative agent, a novel coronavirus, was detected, and being similar to the SARS virus, was named SARS-CoV-2. The virus rapidly spread despite the attempted containment in the city, with over 7000 cases reported in China and 82 cases (in 18 countries) outside of China by the end of January 2020, when the WHO declared a public health emergency. ${ }^{(1)}$

On I I March 2020 the WHO declared COVID-19 a pandemic and South Africa had by that time recorded 13 cases, all of which had been imported. As of 17 June 2020, South Africa has recorded over 80000 cases and more than I 600 deaths.(2)

COVID-19 is mainly a respiratory illness and lung imaging plays an important role. This was even more evident with the initial low sensitivity of Reverse Transcriptase-Polymerase Chain Reaction (RT-PCR) tests, in addition to the long turnaround time to yield results. A large study of I 014 patients in Wuhan showed that Computed Tomography (CT) had a higher sensitivity (98\%) compared to a RT-PCR test (7I\%).(3) As more and more studies were performed, however, it became evident that $\mathrm{CT}$ had a low negative predictive value in the early stages of the disease and hence societies such as the Society of Thoracic Radiology and the American Society for

\section{ABSTRACT}

Coronavirus disease (COVID-19) started as a cluster of pneumonia cases in December 2019 and has rapidly spread across the world, resulting in millions of infections, and approaching one million deaths. In this short review we describe the most common chest $x$-ray and computed tomography manifestations of the virus as it affects the respiratory system, and make brief references to the common clinical presentations.

SAHeart 2020; 17:276-28।

Emergency Radiology recommended that routine CT screening should not be used for diagnosis. ${ }^{\left({ }^{4}\right)}$ Conversely, an abnormal chest CT is not specific for COVID-19 infection.

Chest X-ray (CXR) and CT lung findings of COVID- 19 infection have now been well described, and will be discussed and described in this pictorial review.

The following are commonly reported findings:

Ground glass opacity

- Consolidation

- Linear opacity

Crazy-paving

- Reversed halo sign

\section{GROUND GLASS OPACITY (GGO)}

GGO is the most commonly reported finding on CXR and CT chest. ${ }^{(3)}$ Abnormalities are more often described as bilateral than unilateral (except in early disease), peripheral more than central in location, but with increasing severity there is notably increased total lung involvement.(3)

Ground glass opacity (GGO) is defined by the Fleischner Society as a "hazy increased lung density with preservation of bronchial and vascular walls".(5) CT is more sensitive for detecting GGO (Figure 2). On the CXR, GGO is seen as peripheral areas of increased density with loss of clarity of bronchovascular markings due to superimposition of structures (Figure I). 
GGO has a wide differential diagnosis, including infection (viral, fungal and less often bacterial), fluid overload, pulmonary haemorrhage and interstitial lung disease (including hypersensitivity pneumonitis and collagen vascular disease). In the setting of HIV, infection due to Pneumocystis jerovicii pneumonia as well as drug toxicity should also be considered.

\section{CONSOLIDATION}

Consolidation is defined as the process whereby fluid or cells replace the alveolar air to such an extent that the lung becomes solid.(5) Consolidation is associated with the air-bronchogram
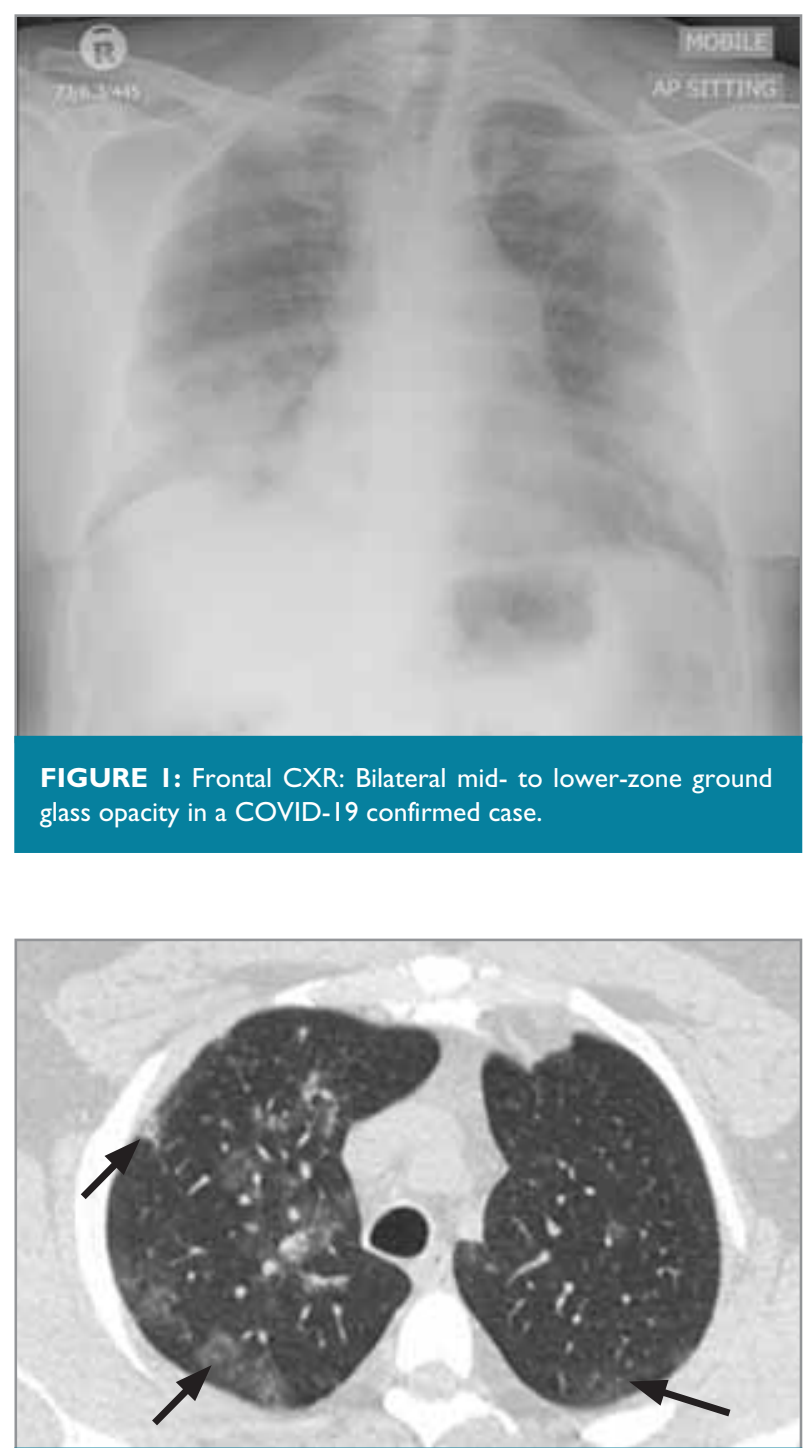

FIGURE 2: Axial CT shows patchy and lobular GGO in a predominantly peripheral distribution in this confirmed COVID-19 patient. GGO can be seen extending into the upper lobes - especially notable on CT. Note the preservation of bronchovascular structures (bronchial wall and vessels can be seen through the lesions), typical for GGO.

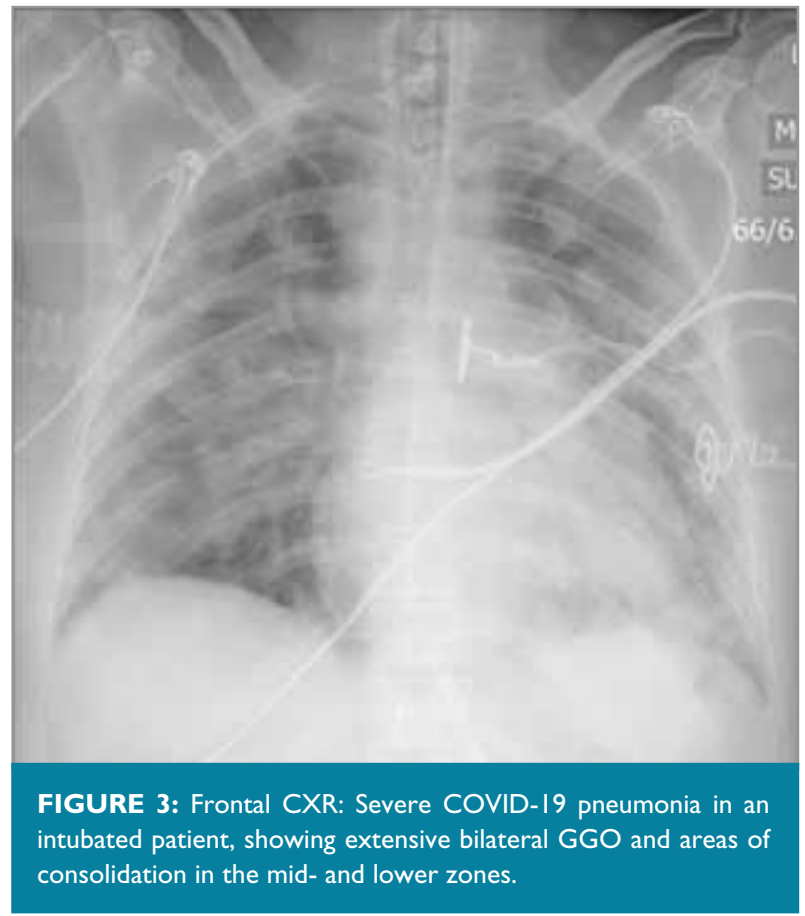

sign, which is due to air-filled bronchi being outlined by airless or densely opacified lung parenchyma. This sign can be seen on CXR and CT and is commonly associated with infectious (bacterial) pneumonia in the acute setting. In COVID-19 pneumonia, consolidation is multifocal, and is often segmental with mid to lower zone preponderance (Figure 3). As the disease progresses, consolidation becomes the more dominant pattern with increasing central lung involvement (Figures 4, 5). ${ }^{(6)}$

\section{LINEAR OPACITY}

Described as lines or band-like, linear opacities can be as a result of atelectasis or interstitial thickening (Figure 6). Linear opacities can be oriented horizontally, obliquely or parallel to the pleural surface and are often longer and thicker than interlobular septa (Figure 7). They can have an arc-shaped morphology in keeping with perilobular opacities, i.e. disease process at the periphery of the secondary lobule (Figure 8), which is well described in organising pneumonia.(5)

\section{CRAZY-PAVING}

Crazy-paving is the appearance of the lung when ground glass opacities are superimposed with reticular opacities, the latter in the form of interlobular septal and intra-lobular interstitial thickening. ${ }^{(5)}$ This finding is commonly seen in other infections such as PCP, in pulmonary oedema, as well as in interstitial lung disease. As COVID-19 disease severity increases, crazypaving (Figure $9 a+9 b$ ) is more frequently seen along with 


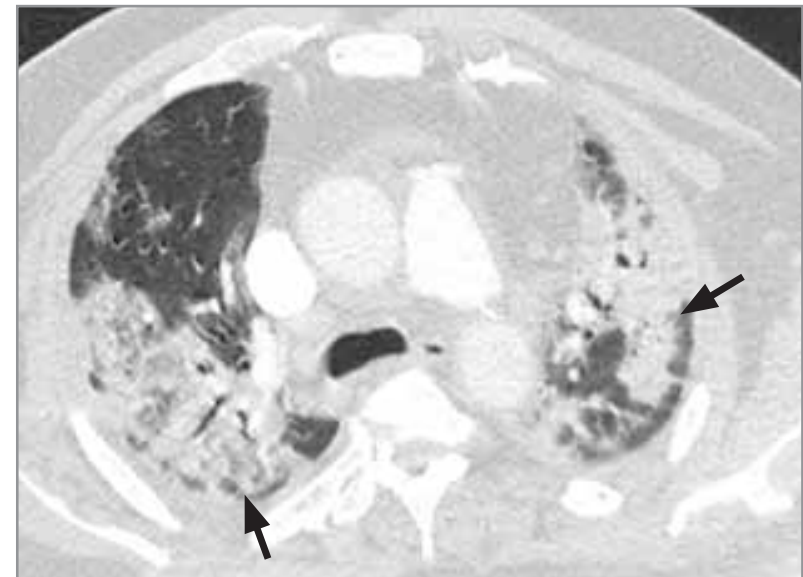

FIGURE 4: Axial CT of a patient with confirmed COVID-19 pneumonia, demonstrating extensive bilateral peripheral and central mixed density opacification with areas of consolidation and air-bronchogram. Areas of sub-pleural sparing are denoted bilaterally (arrow).

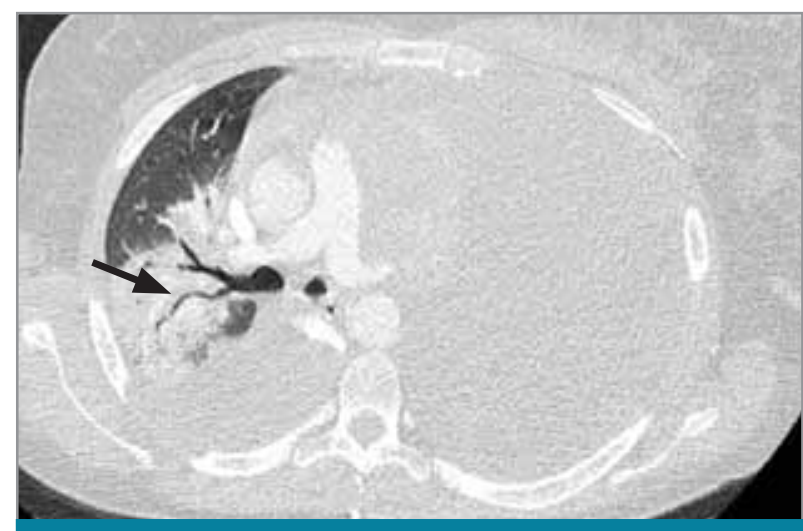

FIGURE 5: Axial CT: Right upper lobe consolidation in a confirmed COVID-19 patient with pre-existing lymphoma (causing a large mediastinal mass and bilateral pleural effusions). An air-bronchogram sign is shown by the arrow.

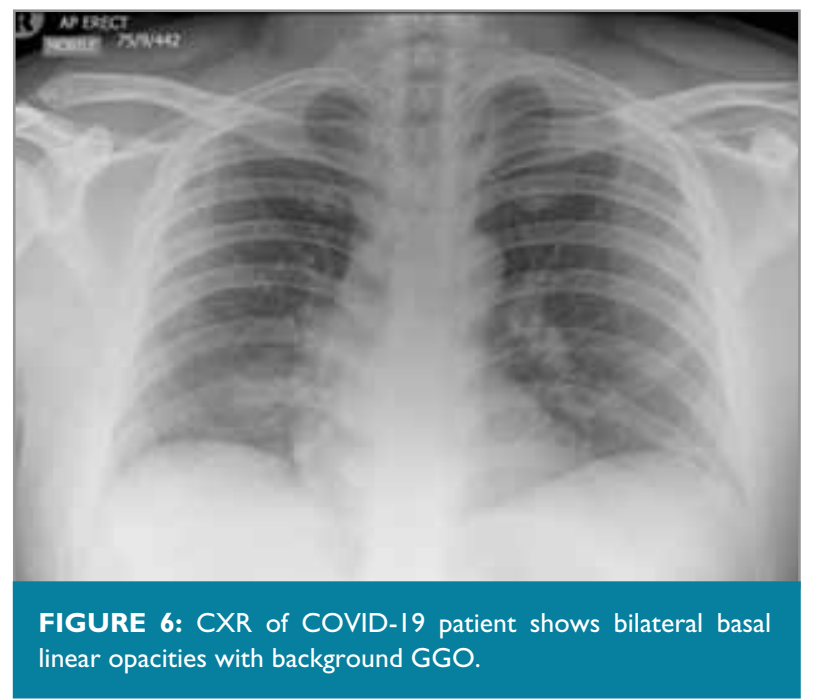

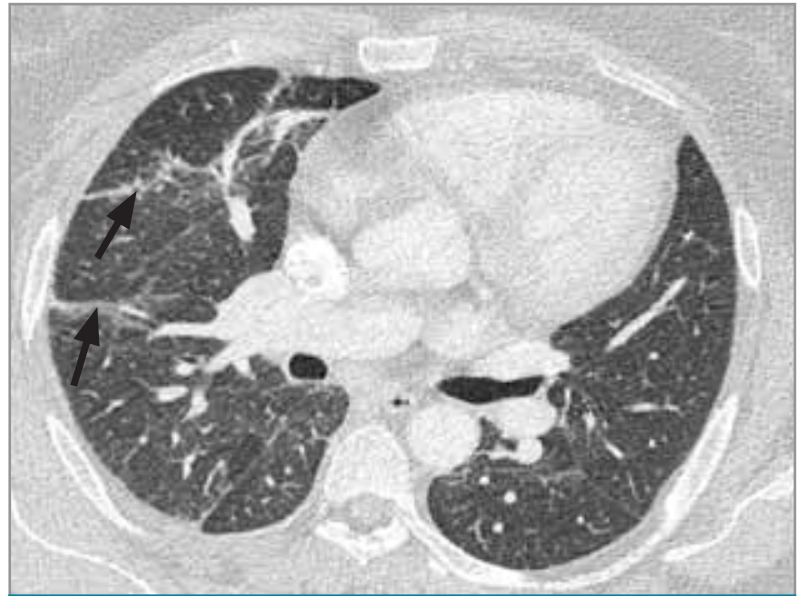

FIGURE 7: Axial CT: Right mid to lower zone linear opacities (arrows) in a COVID-19 confirmed patient.

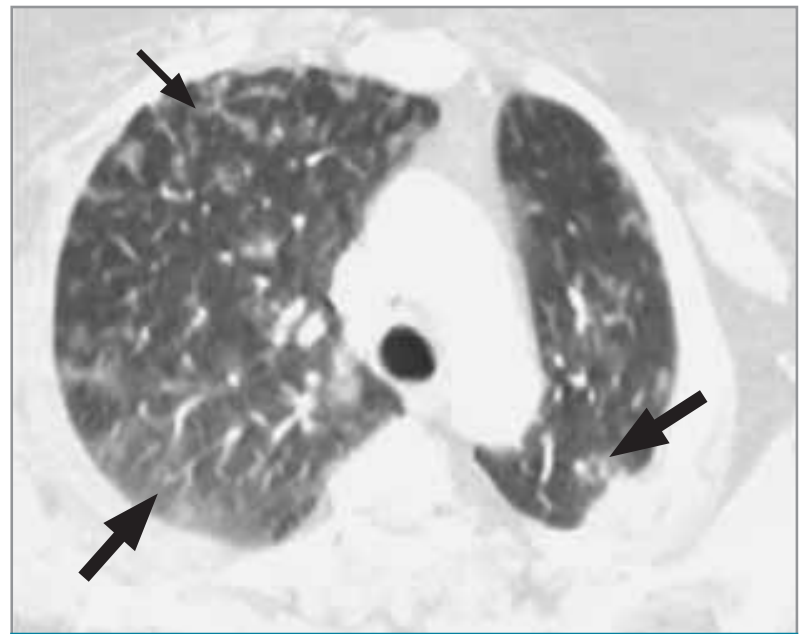

FIGURE 8: Axial CT: Bilateral linear opacities in a perilobular pattern (small arrow), as well as posterior GGO bilaterally (large arrows).

consolidation and air-bronchogram and is associated with a poorer prognosis. ${ }^{(6)}$

\section{REVERSED HALO (ATOLL) SIGN}

The reversed halo sign is defined as a focal rounded area of ground glass opacification surrounded by a rim (complete or incomplete) of consolidation. ${ }^{(5)}$ This has been likened to a coral reef or "atoll", hence the name and it has been described in patients with organising pneumonia and now in COVID-19 pneumonia. It typically develops during the later stages of the disease in areas with nodular opacities (Figure 10a) or consolidations. (7) In addition, a perilobular morphology has also been described in many patients with confirmed COVID-19 pneumonia (Figure IOb), with arc-like linear opacities conforming to the periphery of the secondary pulmonary lobule. (7) 

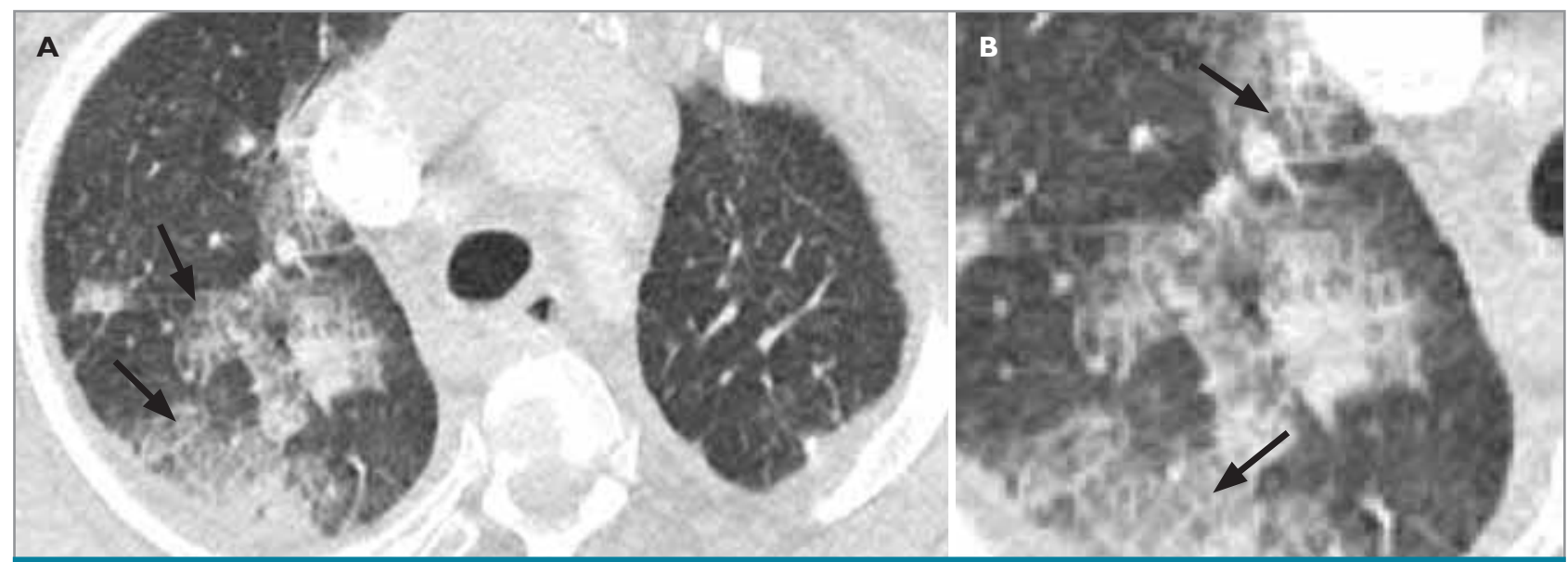

FIGURE 9a and b: Axial CT. Figure 9a demonstrates patchy ground glass opacities superimposed with interstitial thickening, resulting in a crazy-paving pattern (arrows). Zoomed image (Figure 9b) of the right upper lobe with crazy-paving.

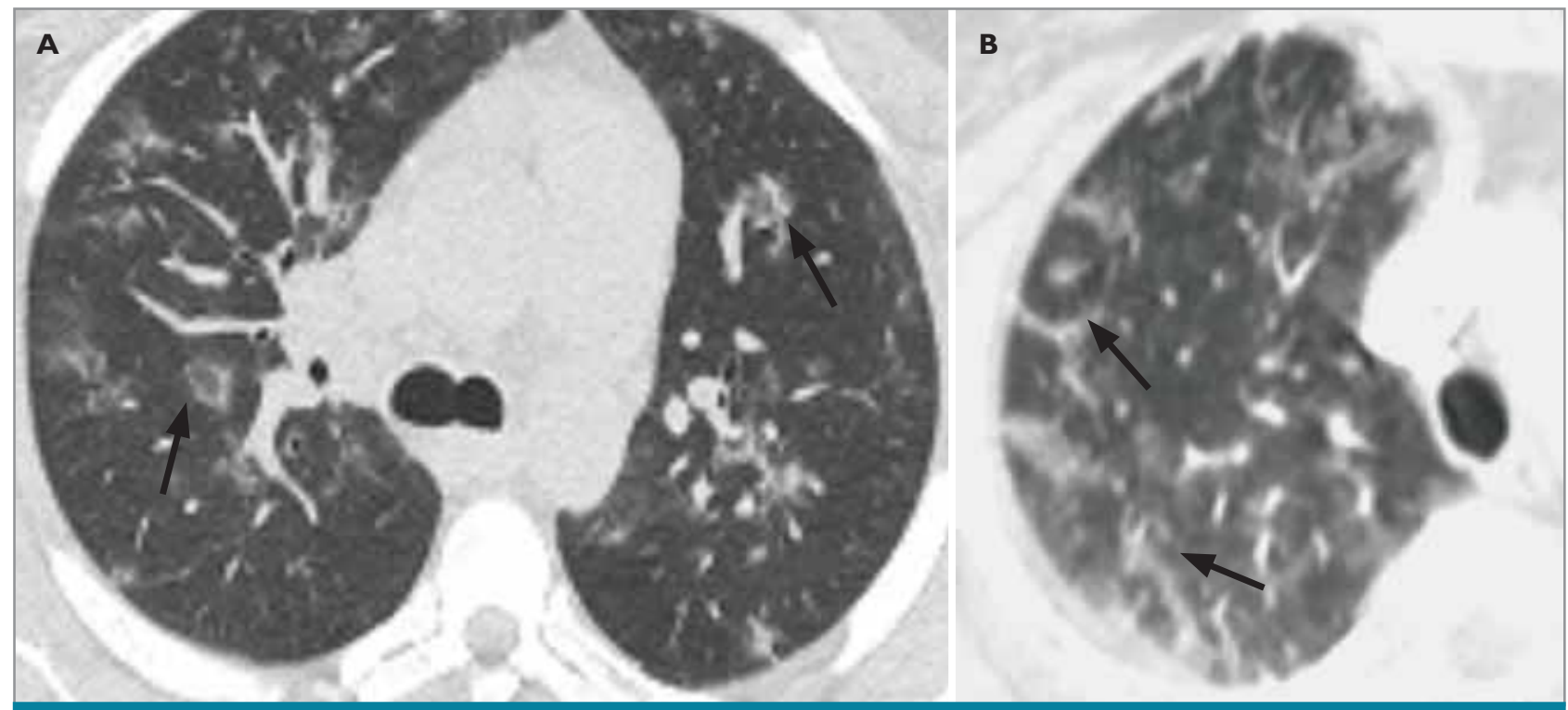

FIGURE IOa and b: Reversed halos can be seen in these 2 patients with confirmed COVID-19 pneumonia (arrows). Areas of central low density GGO are surrounded by a rim of consolidation.

Less commonly reported findings are:
Discrete nodules with/without tree-in-bud
- Pleural effusions
- Lymphadenopathy
- Cavitation

\section{DISCRETE NODULES}

Discrete nodules are less commonly described, if at all reported in COVID-19 pneumonia (Figure 1I). More often, disease detected in the early stage has been described as nodular, sometimes solitary, with subsequent development of bilateral multifocal more extensive changes. ${ }^{(8)}$

\section{PLEURAL EFFUSIONS}

Typically occurring in the later stage of the disease in a small number of patients, pleural effusions are a less typical finding in COVID-19 pneumonia. When present, pleural effusions have been associated with a poor prognosis. ${ }^{(6)}$ The presence of a pleural effusion should raise suspicion for acute pulmonary embolism, as would be the case of non-COVID patients, and this diagnosis must always be considered when there is an acute clinical deterioration (Figure 12). Research has shown that earlier detection of pulmonary embolism may improve outcome with resultant earlier commencement of therapeutic dose anti-coagulation. ${ }^{(9)}$ A significantly elevated D-dimer level 

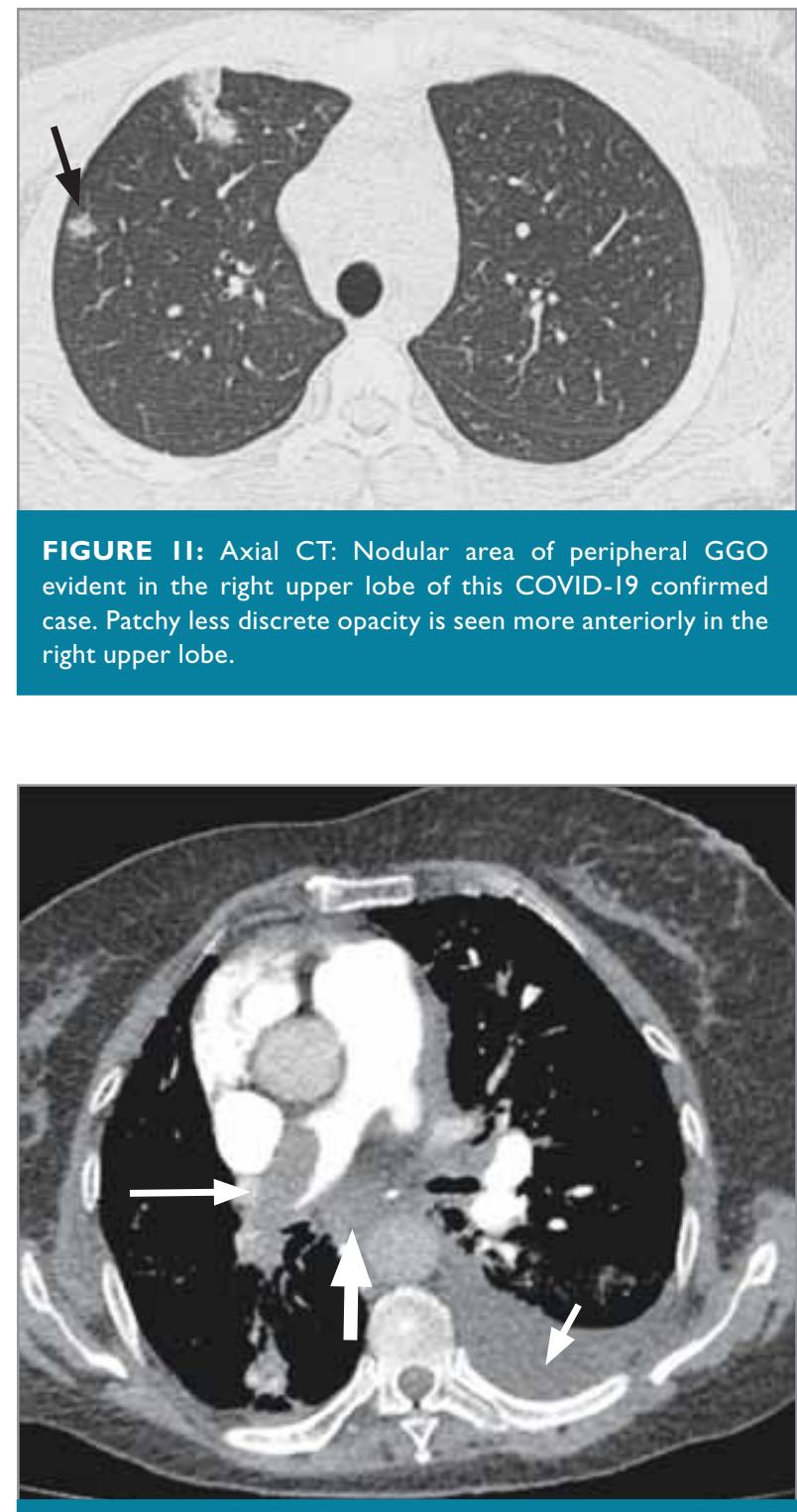

FIGURE 12: Axial CT: COVID-19 confirmed patient complicated by extensive bilateral pulmonary embolism, worse on the right (long arrow). Associated small left pleural effusion (short arrow) and subcarinal lymphadenopathy (fat arrow).

has also been associated with poor outcome. ${ }^{(9)}$ In patients with myocardial involvement, pleural effusions are a sign of cardiac failure and fluid overload. Pre-existing pleural disease should also be considered (Figure 13) in immune-compromised patients, including infection and metastatic involvement.

\section{LYMPHADENOPATHY}

Lymphadenopathy is a less common finding in COVID-19 pneumonia patients (Figure 12) compared to non-COVID pneumonia. ${ }^{(7)}$ In South Africa, a country with a high HIV prevalence of $20.4 \%$ amongst adults, ${ }^{(10)}$ lymphadenopathy

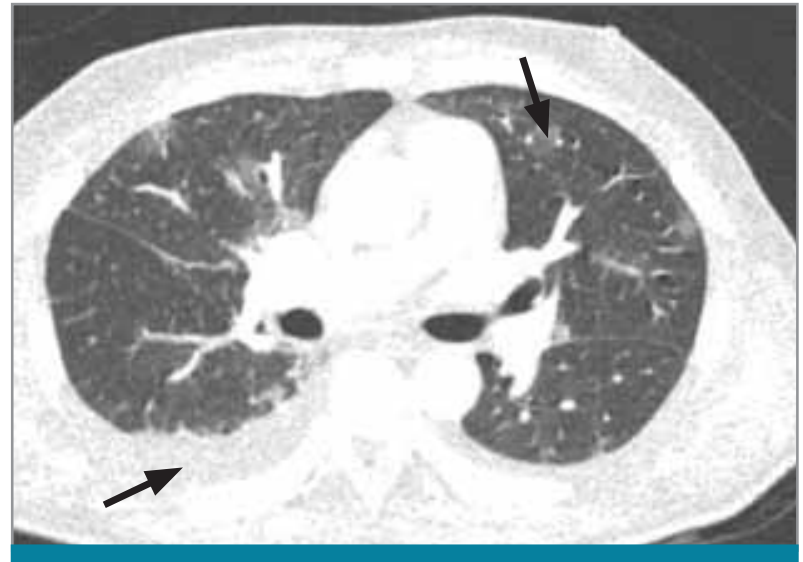

FIGURE 13: Axial CT: Bilateral peripheral GGO with small pleural effusions in a patient diagnosed with leukaemia and post stem cell transplant.

should raise suspicion of other pre-existing infectious aetiologies such as mycobacterial infection and malignancy such as lymphoma.

\section{CAVITATION}

Cavitation is described as (intralesional) areas of gas or lucency within airspace opacification. . $^{(5)}$ This is an uncommon finding in COVID-19 infection, but has been described in an early case series. ${ }^{(1)}$ We have not had any cases showing cavitation.

\section{CONCLUSION}

No CT finding is pathognomonic of SARS-CoV-2 infection. In fact, the commonest finding in patients with confirmed COVID-19 pneumonia is GGO,(3) which is also the most commonly described finding in other viral pneumonias such as influenza and SARS amongst others. Clinical-radiologicalvirological correlation is important, however, and in the presence of a pandemic this should be the most likely diagnosis in the radiological report.

In a confirmed COVID-19 pneumonia patient who has persistent hypoxia, findings can include the signs associated with an organising pneumonia such as a perilobular pattern and the reversed halo sign. Early reports of significant clinical improvement following commencement of steroid treatment have been noted and imaging follow-up of these patients will be essential to evaluate for infection resolution as opposed to pulmonary fibrosis as a sequelae.

Pulmonary thrombo-embolism should be considered earlier rather than later during clinical deterioration, ${ }^{(12)}$ and correlation with D-dimer levels with/without CT pulmonary angiography should be used to aid management. ${ }^{(9)}$ 
Lastly, in our HIV-endemic population, the presence of preexisting lung disease, especially pulmonary tuberculosis, is high. In patients with low CD4 counts (<200), Pneumocystis pneumonia must always be considered in the differential diagnosis of GGO and hypoxia. Chest radiographic interpretation can be complicated and a low threshold for CT of the chest is recommended for more accurate evaluation and especially to exclude other conditions.

\section{Conflict of interest: none declared.}

\section{REFERENCES}

I. WHO.int/news-room2020 (17 July 2020). Available from: https://www.who. int/emergencies/diseases/novel-coronavirus-2019/interactive-timeline.

2. COVID-19 South Africa Dashboard (Internet). 2020 (cited 17 July 2020), Available from: Datastudio.google.com

3. Ai T, Yang Z, Hou H, Zhan C, Chen C, Lv W, et al. Correlation of chest CT and RT-PCR testing for coronavirus disease 2019 (COVID-19) in China: A Report of 1014 Cases. Radiology. 2020;296(2):E32-E40.

4. STR/ASER COVID-19 Position Statement March II, 2020. (press release). II March 20202020

5. Hansell DM, Bankier AA, MacMahon H, McLoud TC, Muller NL, Remy J. Fleischner Society: Glossary of terms for thoracic imaging. Radiology. 2008;246(3):697-722.

6. Tabatabaei $\mathrm{SMH}$, et al. Computed tomographic features and short-term prognosis of coronavirus disease 2019 (COVID-19) Pneumonia: A singlecenter study from Kashan, Iran. Radiology: Cardiothoracic Imaging. 2020:p. e200I30.

7. Wang Y, Dong C, Hu Y, Li C, Ren Q, Zhang X, et al. Temporal changes of CT findings in 90 patients with COVID- 19 pneumonia: A Longitudinal Study. Radiology. 2020;296(2):E55-E64.

8. Yu M, Xu D, Lan L, Tu M, Liao R, Cai S, et al. Thin-section chest CT imaging of coronavirus disease 2019 Pneumonia: Comparison between patients with mild and severe disease. Radiology: Cardiothoracic Imaging. 2020; 2(2):e200126.

9. Bompard F, Monnier H, Saab I, Tordjman M, Abdoul H, Fournier L, et al. Pulmonary embolism in patients with COVID-19 pneumonia. Eur Respir J. 2020;56(I).

10. AIDSINFO internet: UNAIDS; 2019 (cited 2020 July 2020). Available from: https://www.unaids.org/en/regionscountries/countries/southafrica.

11. Kong W, Agarwal PP. Chest imaging appearance of COVID-19 Infection. Radiology: Cardiothoracic Imaging. 2020;2(1):e200028.

12. "Pulmonary embolism and COVID-19: Early detection led to swifter treatment, researchers say." ScienceDaily: Henry Ford Health System. 2020 (updated 2 June 2020). Available from: www.sciencedaily.com/releases/ 2020/06/200602183408 